\title{
BMJ Open Observational study of administering intranasal steroid sprays by healthcare workers
}

\author{
Marielle de Boer, ${ }^{1}$ Corine Rollema (D) , ${ }^{2}$ Eric van Roon, ${ }^{3}$ Tjalling de Vries (D) ${ }^{1}$
}

To cite: de Boer M, Rollema C, van Roon $\mathrm{E}$, et al. Observational study of administering intranasal steroid sprays by healthcare workers. BMJ Open 2020;10:e037660. doi:10.1136/ bmjopen-2020-037660

- Prepublication history for this paper is available online. To view these files, please visit the journal online (http://dx.doi org/10.1136/bmjopen-2020037660).

Received 11 February 2020 Revised 22 April 2020 Accepted 19 July 2020

\section{Check for updates}

(C) Author(s) (or their employer(s)) 2020. Re-use permitted under CC BY-NC. No commercial re-use. See rights and permissions. Published by BMJ.

${ }^{1}$ Paediatrics, Medical Center Leeuwarden, Leeuwarden, The Netherlands

${ }^{2}$ Clinical Pharmacy and Pharmacology, Medical Center Leeuwarden, Leeuwarden, The Netherlands

${ }^{3}$ Pharmacotherapy and

Pharmaceutical Care, University of Groningen, Groningen, UK

Correspondence to Dr Tjalling de Vries; tjalling.de.vries@znb.nl

\section{ABSTRACT}

Objectives Patients with allergic rhinitis receive their information about administering intranasal corticosteroid sprays (INCS) from healthcare workers. Since the majority of patients does not administer these sprays correctly, we investigated whether healthcare workers know how to administer INCS.

Settings We studied participants at their working place: pharmacy, outpatient clinic or general practitioner centre for emergencies.

Participants Pharmacist assistants, general practitioners, paediatricians and ear nose throat doctors.

Design Observational study. All the participants demonstrated the administration technique with a spray device filled with water.

Primary outcome Number of steps of administration of INCS based on the established INCS protocol.

Secondary outcome Number of five steps are labelled essential to obtain optimal distribution of the medication. Results Among the 75 participants, none performed all the steps correctly. The median of correctly performed steps in the protocol was 14 out of 29 . A significantly better result was found among the pharmacist assistants. The essential steps were performed by 27 out of the 75 participants (36\%).

Conclusion The majority of healthcare workers does not know how to administer INCS correctly. Patients could, therefore, receive incorrect and non-uniform instructions. The education of healthcare workers on how to administer INCS correctly may be an option for improvement.

\section{INTRODUCTION}

Allergic rhinitis is a common chronic disease, with a prevalence ranging from $10 \%$ to $40 \%$ worldwide. ${ }^{1}$ The disease mostly affects people between 5 and 45 years old, with a peak incidence between 19 and 24 years old. ${ }^{2}$ In addition to allergen avoidance, the management of allergic rhinitis consists of either antihistamines, intranasal corticosteroids or a combination of both. ${ }^{34}$ When persistent symptoms occur, intranasal corticosteroid sprays (INCS) are the treatment of choice. ${ }^{56}$

Studies suggest that the administration technique for INCS is associated with efficacy, adverse events and compliance. ${ }^{4} 7$ For example, it was found that the optimal

\section{Strengths and limitations of this study}

This is the first study to observe healthcare workers administering intranasal corticosteroid sprays.

- We conducted this study in a small research group, consisting of 75 healthcare workers.

- The administration technique was scored in a simple and robust way by the same investigator.

- We used a protocol for correct administration based on the literature and patient information leaflets, however, not all steps are based on firm scientific evidence.

administration technique of INCS is spraying with a contralateral spray technique, pointing the nozzle away from the septum, because spraying towards the septum causes more nosebleeds than the recommended approach. ${ }^{7-9}$ Furthermore, the head should be kept in a neutral position, and it is important to breathe in calmly while spraying. This technique provides the best distribution of the medication. ${ }^{10-12}$

In a recent study, we observed the method of administration among patients and found that most $(94 \%)$ did not take their INCS as described in the patient information leaflets (PILs). ${ }^{13}$ Moreover, the available PILs are currently incomplete and non-uniform. ${ }^{14}$ Since patients do not only learn from PILs but can also be instructed by healthcare workers, we wanted to know whether they could demonstrate the administration of INCS correctly. As far as we know, this factor has never previously been studied. Therefore, we investigated the knowledge of healthcare workers regarding administering INCS.

\section{MATERIALS AND METHODS}

Participants and setting

We performed an observational study in the northern part of the Netherlands. The healthcare workers observed included pharmacist assistants, general practitioners (GPs), 
paediatricians and ear nose throat (ENT) physicians. Healthcare workers were excluded if they had been practising their profession for less than a year. Paediatricians and ENT physicians from eight hospitals were approached via email. We approached pharmacist assistants from six pharmacies. GPs were approached while working at the GP centre for emergencies based at the Medical Centre Leeuwarden.

\section{Study design}

When eligible healthcare workers agreed to participate, a face-to-face interview was conducted. All the participants were aware of the aim of the study. To prevent interobserver variation, all interviews and observations were performed by the same researcher $(\mathrm{MdB})$.

During the interviews, the healthcare workers answered a questionnaire containing questions about their experiences prescribing INCS and about how they provide patients with information. Then, the healthcare workers were asked to demonstrate the administration technique with a spray device filled with water. The stages assessed were preparation, administration and cleaning of the INCS (box 1). Assessment of the administration technique was based on the established Dutch INCS protocol. During administration, this protocol was used as a scoring sheet, consisting of 29 steps. One point was given each time the participant performed a step correctly. In the INCS protocol, five steps are labelled as essential. ${ }^{14}$ These steps are considered as essential because they most influence the distribution and efficacy of the medication. The essential steps include shaking the bottle, blowing or rinsing the nose, directing the nebuliser away from the nasal septum, breathing in while simultaneously squirting a spray of mist and exhaling through the mouth.

\section{Statistical analysis}

In the statistical analysis, descriptive statistics were used to answer the question of whether healthcare workers know how to administer INCS. For the comparison of outcomes between populations, a Kruskal-Wallis test was used. Post hoc analysis was performed using a Mann-Whitney $\mathrm{U}$ test. The regional medical ethics committee approved the study protocol. All the participants provided written consent.

\section{Patient and public involvement}

Patients or the public were not involved in the design, or conduct, or reporting or dissemination plans of our research.

\section{RESULTS}

In the period between 11 June 2019 and 18 December 2019, 10 ENT departments, seven paediatrician departments and eight pharmacies were asked to participate in this study. GPs were approached at the GP's emergency centre and asked to participate on the spot. Seventy-five healthcare workers participated in our study (table 1),
Box 1 Assessed steps per stage of the intranasal corticosteroid sprays protocol

\section{Preparation}

1. Discuss the purpose and action of the medication.

2. Take off the dust cap.

3. Firmly shake the bottle. *

4. Place forefinger and middle finger on both sides of the nozzle and place thumb underneath the bottle.

5. Point the nozzle upwards and away from yourself.

6. Squirt a few sprays into the air until you see a cloud of mist.

7. Blow the nose or rinse the nose with saline if the nose is clogged. *

\section{Administration}

8. Place forefinger and middle finger on both sides of the nozzle and place thumb underneath the bottle.

9. A. Keep the head upright and place the nozzle in the nose. B.Use the right hand for spraying in the left nostril and the left hand for spraying in the right nostril.

10. Point the end of the nozzle slightly outwards, away from the centre ridge of the nose.*

11. Close the other nostril with your opposite hand.

12. Squirt a spray of mist in the nose while breathing in.*

13. Breathe out through the mouth. *

14. Repeat steps 8 through 13 for the other nostril.

15. If two sprays per nostril are prescribed, repeat steps 8 through 14 for both nostrils.

16. Replace the dust cap.

Cleaning

17. Wipe the nozzle with a tissue or handkerchief after every use.

18. Extensively wash the nozzle once a week:

19. Take off the dust cap and nozzle.

20. Rinse the dust cap and nozzle with warm water.

21. Shake off water.

22. Air-dry the dust cap and nozzle.

23. Replace the nozzle.

24. Firmly shake the bottle.

25. Squirt a few sprays into the air until you see a cloud of mist.

26. Replace the dust cap.

\section{Points for attention}

27. If the nozzle does not spray properly, perform the cleaning steps. If this does not work, take the spray to the pharmacy. Never puncture the opening.

28. Check the expiry date on the package and the expiry date after opening.

${ }^{\star}$ Essential steps.

none of them performed all the steps correctly. The median of correctly performed steps in the protocol was 14 out of 29 , with a range between 9 and 24 and an IQR of 3.5. For preparing the spray, the median was six out of seven steps. The median for administering the spray was 8 out of 10 steps and the median for cleaning the spray was 1 out of 10 steps. The points for attention, which are taking the spray to the pharmacy when it does not work and checking the expiry date, were not performed by most participants (figure 1). When analysing the differences in total score between the healthcare workers, a significantly higher result was found among pharmacist assistants compared with the other groups (table 2). 
Table 1 Participant characteristics

\begin{tabular}{ll}
\hline Variable & Overall $(\mathbf{n}=\mathbf{7 5})$ \\
\hline Healthcare worker, $\mathrm{n}(\%)$ & \\
\hline Pharmacist assistant & $20(26)$ \\
General practitioner & $20(26)$ \\
Paediatrician & $20(26)$ \\
ENT physician & $15(20)$ \\
Gender, $\mathrm{n}(\%)$ & \\
$\quad$ Male & $27(36)$ \\
Female & $48(64)$ \\
Age (mean), range $(95 \% \mathrm{Cl})$ &
\end{tabular}

Age (mean), range $(95 \% \mathrm{Cl})$

$45.2 \pm 11$

\begin{tabular}{|cc}
\hline Years of professional practice, $\mathrm{n}(\%)$ \\
\hline $1-5$ years & $18(24)$ \\
\hline $5-10$ years & $12(16)$ \\
\hline $10-20$ years & $23(29)$ \\
\hline$>20$ years & $20(27)$ \\
\hline Number of INCS prescriptions per week, $\mathrm{n}(\%)$ \\
\hline 0 times & $19(25)$ \\
\hline$<1$ time & $17(23)$ \\
\hline $1-4$ times & $5(7)$ \\
\hline $4-8$ times & $14(19)$ \\
\hline$>8$ times & $24(32)$ \\
\hline Number of INCS instructions per week, $\mathrm{n}(\%)$ \\
\hline$<1$ time & $27(36)$ \\
\hline $1-4$ times & $9(12)$ \\
\hline $4-8$ times & $15(20)$ \\
\hline$>8$ times & $19(25)$ \\
\hline Number of checks on INCS inhalations per week, $\mathrm{n}(\%)$ \\
\hline$<1$ time & $62(83)$ \\
\hline $1-4$ times & $15(14)$ \\
\hline $4-8$ times & $2(3)$ \\
\hline Use of information material, $\mathrm{n}(\%)$ & $0(0)$ \\
\hline Yes & \\
\hline No & \\
\hline
\end{tabular}

ENT, ear nose throat; INCS, intranasal corticosteroid sprays.

Twenty-seven out of 75 participants $(36 \%)$ performed all the essential steps. Of the essential steps, nose blowing was performed least frequently, being carried out 46 out of 75 times $(61 \%)$. Inhaling while squirting a spray of mist into the open nostril was performed most often: 69 out of 75 times (92\%). Shaking the bottle was done 61 times $(81 \%)$. The nozzle was directed outwards 66 times $(88 \%)$. Exhaling through the mouth was done by 57 participants (76\%) (table 3).

Sixteen participants $(21 \%)$ performed all the preparation steps. The least performed steps during preparation
SCORE CHECKLIST PER STAGE

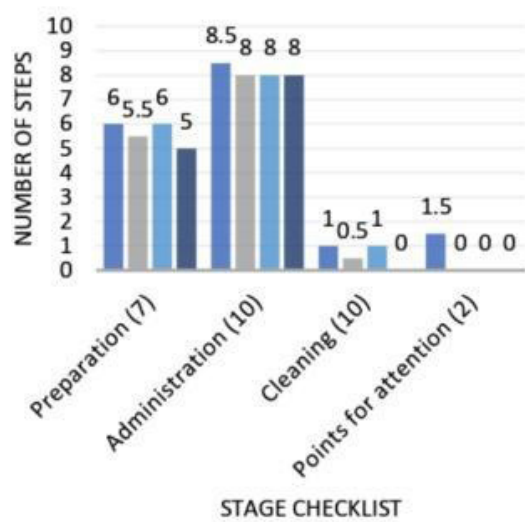

w Pharmacist assistant $=$ General Practitioner

m Paediatrician $\quad$ ENT physician

Figure 1 Median score checklist per stage, per group of healthcare workers. ENT,ear nose throat.

were activating the nozzle by spraying in the air (59\%) and blowing or rinsing the nose $(61 \%)$. Two participants (3\%) performed all the administration steps. Squirting the spray while breathing in $(92 \%)$ and pointing the nozzle outwards $(88 \%)$ were performed by most participants. The most frequent errors in administration were, in order, keeping the other nostril closed, spraying crosswise and keeping the head upright. These steps were performed 33 times (44\%), 43 times (47\%) and 49 times $(65 \%)$, respectively. One participant $(1 \%)$ performed all the cleaning steps, including cleaning the nozzle with a tissue and extensively cleaning the nozzle with water once a week and allowing it to air-dry. Approximately, half the participants $(38 ; 51 \%)$ cleaned the spray with a tissue after every use (table 3 ).

\section{DISCUSSION}

In this study, we found that most healthcare workers involved in the care for patients with allergic rhinitis did not know and could not demonstrate how to administer INCS correctly. None of the healthcare workers performed all the steps of the checklist correctly. The median of the 75 participants was 14 out of 29 well-executed steps. The five essential steps were correctly performed by 27 out of 75 participants (36\%). We found that the pharmacist assistants administered the INCS significantly better than the other healthcare workers, suggesting that pharmacist assistants are most suitable for providing information about INCS.

To the best of our knowledge, the role of healthcare workers in informing patients about the correct administration of INCS has never previously been studied. One recent study found that face-to-face instruction, consisting of both verbal information and a physical demonstration, was most effective in teaching the correct technique for asthma medication. ${ }^{15}$ This finding may apply regarding the correct usage of INCS as well, meaning 
Table 2 Difference in total score checklist

\begin{tabular}{llllll}
\hline Healthcare worker & N & Median & Mean & Mean rank & P value between groups* $^{*}$ \\
\hline Pharmacist assistant & 20 & 16 & 16.2 & $54.58 \dagger$ & 0.001 \\
General practitioner & 20 & 13.5 & 13.3 & 30.98 & 34.85 \\
Paediatrician & 20 & 14 & 13.8 & 29.47 & \\
ENT physician & 15 & 12 & 13.1 & \\
\hline
\end{tabular}

Tested using Kruskal-Wallis test (post hoc Mann-Whitney U).

${ }^{*} \mathrm{p}<0.05$.

†Significant difference compared with general practitioners, paediatricians and ENT physicians.

ENT, ear nose throat.

that it is important that healthcare workers have sufficient knowledge. Knowledge about inhaler technique for asthma and chronic obstructive pulmonary disease has been researched using healthcare workers. These studies reveal similar results to ours; that is, inadequate knowledge regarding the use of inhalers. ${ }^{16}{ }^{17} \mathrm{~A}$ recent study found only $12 \%$ of healthcare workers knew at least three essential steps of the correct metered-dose inhaler technique, and none of the participants performed all the steps correctly. ${ }^{18}$

For the checklist, we used a standardised protocol recently published in Dutch healthcare. Although this protocol is based on the existing literature, a few instruction steps are only marginally substantiated by research data, and we needed to make a few assumptions about the best spray technique (table 4). First, an essential step while preparing for administration is blowing or rinsing the nose. This step is recommended in the literature, although no significantly better distribution of the active substance was found. ${ }^{19}$ Approximately $60 \%$ of the participants performed this step. Next, it was found that an upright position of the head while spraying proved most effective. ${ }^{1020}$ Bending the head backwards can result in the active substance leaking into the pharynx, causing irritation and possibly more systemic uptake. When bending the head forward, the active substance could easily run out again. However, $45 \%$ of the ENT doctors and $10 \%-20 \%$ of the paediatricians, GPs and pharmacist assistants bent their heads forward while breathing in the spray. Their reasoning was that the nasal cavity runs backwards, causing the spray to be sprayed towards the nasal cavity when the head is bent forward. There is a study that confirms this theory. ${ }^{12}$ When administering the nasal spray, the nozzle should be directed away from the nasal septum to prevent nose bleeding and septum perforation. Furthermore, the lateral nasal wall has more cilia than the medial wall, so the distribution of the active substance is better. ${ }^{79}$ We found that this essential step was performed correctly by $88 \%$ of the participants. It is also advised to use the contralateral hand while spraying, because this causes less mechanical irritation, and, thus, fewer side effects and better compliance. ${ }^{7}$ Approximately half the participants $(57 \%)$ performed this step. However, not all these participants recommended this step to their patients, because it is relatively complicated to perform. Another essential step is to breathe in slowly during administration, because the airflow ensures a better contribution of the active substance. A continuous, slow flow has the best effect. Breathing hard or sniffing can lead to increased turbulence in the nose, which can cause the active substance to finish in the pharynx..$^{1121}$ This was the best-performed essential step: $92 \%$ of participants performed this correctly. Only one participant stated that the nasal spray should be completely cleaned with water once a week. Since these nasal sprays are used chronically and daily in many cases, good hygiene is important. However, there is no uniform instruction for cleaning the nasal spray in the package leaflets. This lack is a possible explanation why the healthcare workers did not mention this aspect. Considering that there is little substantiated research about the correct administration technique, and since the published INCS protocol is not widely known, healthcare providers apparently provide instructions according to their own insights. Further research is necessary to substantiate the most effective administration technique for nasal sprays. Currently, it is important to implement the presently available instructions to achieve clarity in the instructions for the administration of nasal sprays.

\section{Strengths and limitations}

This study has a couple of limitations. First, we did not know what outcomes to expect, so we chose to conduct this research with a relatively small research group in a particular region. ${ }^{22}$ Further research must determine to what extent our results apply to other regions and countries. Second, we could only include 15 ENT doctors despite many attempts and visits to their offices. Reasons given were that they were too busy or not interested in participating. Nevertheless, we do not think that the inclusion of five more ENT doctors would affect the outcome significantly. Third, it is possible that healthcare workers only agreed to participate in this study when they felt an affinity with the topic. Greater interest might influence the knowledge of a correct administration technique. Healthcare workers with less affinity would possibly score lower. Given the disappointing results of the studied population, this only highlights the fact that the healthcare workers' knowledge about the correct administration technique must improve. Finally, we studied the 
Table 3 Number of well-executed steps protocol

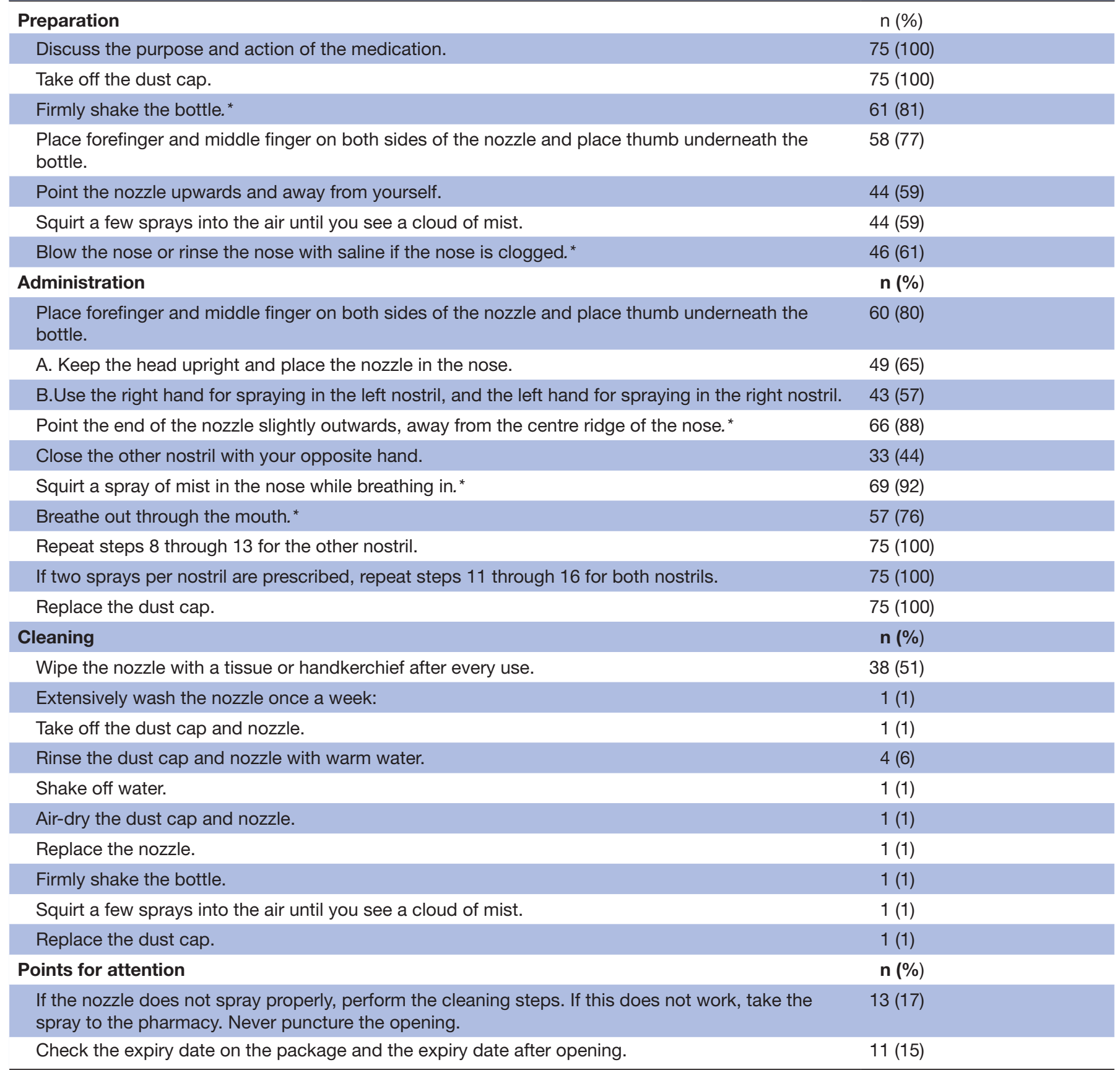

*Essential steps.

knowledge of healthcare workers, not the actual instruction patients receive from them.

The strengths of this study are as follows: since various hospitals, general practices and pharmacies were included, the results are a good reflection of the population that both prescribes and informs about INCS. All our interviews were conducted by the same researcher, so no interobserver variation occurred. We used a simple scoring system, making the scores less sensitive to errors.

This research only studied healthcare workers' knowledge of the administration technique. Studying whether the knowledge of a healthcare worker ultimately affects the correct spray technique, and, thus, the efficacy of INCS in patients is recommended.

\section{CONCLUSION}

The majority of healthcare workers does not know how to administer an INCS correctly. This lack of knowledge may prevent them from being able to provide adequate instructions to their patients. Clear arrangements should be made regarding who provides these instructions and what these instructions include. Healthcare workers should continually update their knowledge regarding the 
Table 4 Scientifically and non-scientifically based steps in the corticosteroid nasal spray protocol

\section{Scientifically based steps}

Blow the nose or rinse the nose with saline if the nose is clogged. $^{19}$

- Keep the head upright. ${ }^{1020}$

Use the right hand for spraying in the left nostril and the left hand for spraying in the right nostril. ${ }^{12}$

- Point the end of the nozzle slightly outwards, away from the centre ridge of the nose.

Squirt a spray of mist in the nose while breathing in.

\section{Non-scientifically based steps}

Firmly shake the bottle.

Squirt a few sprays into the air until you see a cloud of mist.

Close the other nostril with your opposite hand.

\author{
Breathe out through the mouth.
}

Wipe the nozzle with a tissue or handkerchief after every use.

Extensively wash the nozzle once a week. correct spray technique. The established INCS protocols can be used to address this issue.

Acknowledgements We thank the healthcare workers studied for their time and effort in this study.

Contributors MdB was involved in developing the study protocol, interviewed the participants, analysed the data and wrote the first draft. CR was involved in developing the study protocol, analysing the data and in the process of writing. EvR was involved in developing the study protocol and the process of writing. TdV had the original idea, was involved in developing the study protocol, analysing the data and the process of writing. All authors read the final version and agree.

Funding The authors have not declared a specific grant for this research from any funding agency in the public, commercial or not-for-profit sectors.

Competing interests All authors have completed the ICMJE uniform disclosure form at www.icmje.org/coi_disclosure.pdf and declare: no support from any organisation for the submitted work; no financial relationships with any organisations that might have an interest in the submitted work in the previous 3 years; no other relationships or activities that could appear to have influenced the submitted work.

Patient consent for publication Not required.

Provenance and peer review Not commissioned; externally peer reviewed.

Data availability statement Data are available upon reasonable request. Data statement section, technical appendix, statistical code and dataset available from the authors.

Open access This is an open access article distributed in accordance with the Creative Commons Attribution Non Commercial (CC BY-NC 4.0) license, which permits others to distribute, remix, adapt, build upon this work non-commercially, and license their derivative works on different terms, provided the original work is properly cited, appropriate credit is given, any changes made indicated, and the use is non-commercial. See: http://creativecommons.org/licenses/by-nc/4.0/.

\section{ORCID IDs}

Corine Rollema http://orcid.org/0000-0002-7701-3884

Tjalling de Vries http://orcid.org/0000-0003-0940-1719

\section{REFERENCES}

1 Bousquet P-J, Leynaert B, Neukirch F, et al. Geographical distribution of atopic rhinitis in the European community respiratory health survey I. Allergy 2008;63:1301-9.

2 Nederlands Huisartsen Genootschap. Samenvattingskaart M48. Allergische en niet-allergische rhinitis. Herziening, 2018. Available: https://www.nhg.org/standaarden/samenvatting /allergische-en-nietallergische-rinitis
3 Platts-Mills TAE. Allergen avoidance in the treatment of asthma and allergic rhinitis, 2019. Available: https://www.uptodate.com [Accessed 12 May 2019].

4 deShazo RD, Kemp SF. Pharmacotherapy of allergic rhinitis, 2018. Available: https://uptodate.com [Accessed 12 May 2019].

5 Fokkens WJ, Lund VJ, Hopkins C, et al. European position paper on rhinosinusitis and nasal polyps 2020. Rhinology 2020;58:1-464.

6 Karatzanis A, Chatzidakis A, Milioni A, et al. Contemporary use of corticosteroids in rhinology. Curr Allergy Asthma Rep 2017;17:11.

7 Ganesh V, Banigo A, McMurran AEL, et al. Does intranasal steroid spray technique affect side effects and compliance? results of a patient survey. J Laryngol Otol 2017;131:991-6.

8 Chong LY, Head K, Hopkins C, et al. Intranasal steroids versus placebo or no intervention for chronic rhinosinusitis. Cochrane Database Syst Rev 2016;4:CD011996.

9 Benninger MS, Ahmad N, Marple BF. The safety of intranasal steroids. Otolaryngol Head Neck Surg 2003;129:739-50.

10 Benninger MS, Hadley JA, Osguthorpe JD, et al. Techniques of intranasal steroid use. Otolaryngol Head Neck Surg 2004;130:5-24.

11 Garlapati RR, Lee HP, Chong FH, et al. Indicators for the correct usage of intranasal medications: a computational fluid dynamics study. Laryngoscope 2009;119:1975-82.

12 Tay SY, Chao SS, Mark KTT, et al. Comparison of the distribution of intranasal steroid spray using different application techniques. Int Forum Allergy Rhinol 2016;6:1204-10.

13 Rollema C, van Roon EN, de Vries TW. Inadequate quality of administration of intranasal corticosteroid sprays. J Asthma Allergy 2019;12:91-4.

14 Rollema C, van Roon EM, Schilder AG, et al. Evaluation of instructions in patient information leaflets for the use of intranasal corticosteroid sprays: an observational study. BMJ Open 2019;9:e026710.

15 Bosnic-Anticevich SZ, Sinha H, So S, et al. Metered-Dose inhaler technique: the effect of two educational interventions delivered in community pharmacy over time. J Asthma 2010;47:251-6.

16 Self TH, Arnold LB, Czosnowski LM, et al. Inadequate skill of healthcare professionals in using asthma inhalation devices. $J$ Asthma 2007;44:593-8.

17 Plaza V, Giner J, Rodrigo GJ, et al. Errors in the use of inhalers by health care professionals: a systematic review. J Allergy Clin Immunol Pract 2018;6:987-95.

18 Adeniyi BO, Adebayo AM, llesanmi OS, et al. Knowledge of spacer device, peak flow meter and inhaler technique (MDIs) among health care providers: an evaluation of doctors and nurses. Ghana Med J 2018;52:15-21.

19 Smith KA, Rudmik L. Delivery of topical therapies. Adv Otorhinolaryngol 2016;79:114-20.

20 Jang TY, Kim YH. Recent updates on the systemic and local safety of intranasal steroids. Curr Drug Metab 2016;17:992-6.

21 Milgrom H, Bender B. Adverse effects of medications for rhinitis. Ann Allergy Asthma Immunol 1997;78:439-46.

22 Hertzog MA. Considerations in determining sample size for pilot studies. Res Nurs Health 2008;31:180-91. 